\title{
Fatores Prognósticos e Sobrevida no Adenocarcinoma Primário de Reto
}

\section{Prognostic Factors and Survival in Primary Rectal Adenocarcinoma}

\author{
HELOISA GUEDES MÜSSNICH ${ }^{1}$, LUIS FERNANDO MOREIRA ${ }^{2}$, PEDRO GUS $^{3}$, MARCELO PIMENTEL ${ }^{4}$, TATIANA \\ SIMON $^{4}$, MARCELO BELLON DOS SANTOS $^{4}$
}

\begin{abstract}
${ }^{1}$ Coloproctologista, Mestranda do Curso de Pós-Graduação em Medicina: Cirurgia, Faculdade de Medicinal Universidade Federal do Rio Grande do Sul (FAMED/UFRGS), Porto Alegre, RS; ${ }^{2}$ Co-orientador, Professor Doutor do Curso de Pós-Graduação em Medicina: Cirurgia FAMED/UFRGS, Porto Alegre, RS; ${ }^{3}$ Orientador, Professor Titular, Livre-docente em Coloproctologia; ${ }^{4}$ Médico, Bolsista de Iniciação científica, FAMED/ UFRGS, Porto Alegre, RS.
\end{abstract}

\begin{abstract}
MÜSSNICH HG; MOREIRA LF; GUS P; PIMENTEL M; SIMON T; SANTOS MB. Fatores Prognósticos e Sobrevida no Adenocarcinoma Primário de Reto. Rev bras Coloproct, 2008;28(1): 062-071.

RESUMO: Objetivo: Avaliar a sobrevida e fatores clínico-patológicos relacionados ao adenocarcinoma de reto. Metodologia: Foram revisados 112 prontuários de pacientes com adenocarcinoma de reto quanto a: idade, sexo, antígeno carcino-embriônico, curabilidade da cirurgia, seguimento, recidiva, sobrevida e histopatologia do tumor. Para análise da sobrevida, utilizou-se o método de Kaplan-Meyer. Nas análises bivariada e estratificada, $\boldsymbol{P}<\mathbf{0 , 0 5}$ foi significativo. No modelo multivariado, utilizou-se um IC de 90\%. Resultados: O seguimento mediano foi de 35,27 meses (14,5 - 57,63). A sobrevida em 5 anos foi de $51 \%$. Sessenta e quatro pacientes $(57 \%)$ apresentaram recidiva; 45 (40\%) faleceram da neoplasia; $68 \%$ dos tumores estendiam-se até os tecidos perirretais; e 67 pacientes tinham linfonodos positivos (30\% em cada, N1 e N2). Quatorze pacientes eram estágio D; 55, C1 e C2; 15, B2; e 28, B1 e A. O risco de óbito aumentou entre os casos com: estágios avançados, tumores mais invasivos e menos diferenciados, envolvimento linfonodal (N2>N1) e recidiva. A classificação de Dukes e a diferenciação tumoral foram fatores prognósticos independentes, bem como a penetração do tumor na parede retal e o comprometimento linfonodal, quando excluída a classificação histopatológica. Conclusão: Além da diferenciação tumoral, os fatores prognósticos identificados correspondem aos níveis dos sistemas de estadiamento vigentes.
\end{abstract}

Descritores: Adenocarcinoma de reto, fatores prognósticos, classificação histopatológica, recidiva, sobrevida.

\section{INTRODUÇÃO}

O câncer colorretal é a terceira neoplasia mais freqüente no mundo ocidental ${ }^{(1)}$, sendo que o reto é atingido em 30 a $57 \%$ dos $\operatorname{casos}^{(2,3)}$. Mais de $50 \%$ dos pacientes têm tumores avançados no momento do diagnóstico, e a sobrevida em 5 anos se mantém em torno de $50 \%$ em todo o mundo ${ }^{(4-7)}$. Embora um grande número de estudos tenha avaliado vários parâmetros clínicos, patológicos e moleculares em relação ao prognóstico, até o momento, o estadiamento clinicopatológico das lesões, obtido do espécime cirúrgico na ressecção do tumor primário, constitui a informação prognóstica mais importante disponível para esses pacientes ${ }^{(8-12)}$.

O presente estudo tem como objetivo avaliar a sobrevida e os fatores clinicopatológicos relacionados aos tumores de reto em nosso meio.

\section{PACIENTES E MÉTODOS}

Os prontuários de 112 pacientes com adenocarcinoma primário e único de reto médio ou distal 
(de 3 a $12 \mathrm{~cm}$ da margem anal) submetidos à cirurgia eletiva no Serviço de Coloproctologia do Hospital de Clínicas de Porto Alegre, no período de 1988 a 1995, foram revisados retrospectivamente quanto a: idade, sexo, antígeno carcino-embriônico (CEA) pré-operatório, seguimento (até o término da coleta de dados, em 1998), recidiva locorregional ou à distância, sobrevida e histopatologia do tumor (diferenciação celular, grau de penetração na parede, comprometimento linfonodal, produção de muco e comprometimento de margens cirúrgicas). Foram excluídos os casos com lesões sincrônicas, metacrônicas ou simultâneas de outros órgãos, com doenças intestinais associadas ou tratamentos fora da rotina do referido Serviço (retocolite ulcerativa, radioterapia pré-operatória, cirurgia sem ressecção intestinal ou realizada em outro serviço). Os tumores foram classificados, a partir dos laudos anatomopatológicos do Serviço de Patologia do referido hospital, segundo o sistema de estadiamento de Dukes modificado por Astler-Coller, e comparados ao sistema TNM.

O seguimento dos pacientes foi avaliado através dos exames de estadiamento disponíveis nos prontuários (radiografia de tórax, ecografia ou tomografia abdominal, dosagem sérica de CEA e endoscopia digestiva baixa - retossigmoidoscopia ou colonoscopia), ou através do contato com os próprios pacientes ou seus familiares. Os pacientes com doença metastática no momento do diagnóstico foram excluídos da análise de recidiva.

Para permitir tratamento estatístico adequado, em virtude do número reduzido de pacientes em alguns grupos, os estágios da classificação de Dukes modificada por Astler-Coller foram distribuídos em subgrupos, de acordo com o prognóstico e a perspectiva terapêutica atual: subgrupos 1 (A e B1), 2 (B2 e C1) e 3 (C2 e D). O coeficiente de correlação de Kendall foi utilizado para comparação dos sistemas de estadiamento. A sobrevida foi avaliada pelo método de Kaplan-Meyer. Nas análises bivariada e estratificada, um valor de $P$ $<0,05$ foi considerado significativo. No modelo multivariado, utilizou-se um IC de $90 \%(=0,10)$.

\section{RESULTADOS}

As características clínicas dos pacientes estão resumidas na Tabela 1. O CEA pré-operatório foi avaliado em 78,6\% dos casos, e variou de $0,4 \mathrm{ng} / \mathrm{dl}$ a $658 \mathrm{ng} /$ dl, com mediana de 4,75 (2,20 - 13,45). A amputação

abdominoperineal de reto foi realizada em 63 pacientes $(56,3 \%)$, e a retossigmoidectomia, com ou sem anastomose, no restante dos casos. Sessenta e quatro pacientes (57\%) apresentaram recidiva tumoral, sendo $14,3 \%$ locorregional, $32,1 \%$ à distância e $10,7 \%$ simultâneas. O tratamento complementar adjuvante foi realizado em $19,3 \%$ dos casos, e o paliativo, em 32 pacientes $(28,6 \%)$.

O tempo mediano de seguimento foi de 35,27 meses $(14,5$ - 57,63), sendo realizado em $90 \%$ dos casos. Quarenta e cinco pacientes foram a óbito pela doença neoplásica no período de seguimento $(40,2 \%)$, totalizando uma sobrevida, relacionada à doença, de $51 \%$ em 5 anos (Figura 1).

A proporção de sobrevida foi significativamente menor à medida que avançavam os estágios histopatológicos da doença (Figura 2; $P<0,001$ ).

As características anatomopatológicas dos tumores estão representadas na Tabela 2.

$\mathrm{O}$ adenocarcinoma moderadamente diferenciado foi o mais freqüente, em 82 pacientes $(73,2 \%)$. A maior parte dos tumores $(68,2 \%)$ estendia-se até os tecidos perirretais (T3 e T4), e apenas 3,8\% dos pacientes apresentaram lesões restritas à mucosa (T1). A média de linfonodos isolados foi de $14,4 \pm 10,25$. Sessenta e sete pacientes $(62,6 \%)$ tinham linfonodos positivos, sendo 33 deles com 1 a 3 linfonodos comprometidos (N1), e 34 com mais de 3 linfonodos comprome-

Tabela 1 - Características clínicas dos pacientes estudados.

\begin{tabular}{lcc}
\hline Característica & \multicolumn{2}{c}{ Medida descritiva } \\
\hline Idade (anos) & $62,03 \pm 14,37$ & $(19-86)$ \\
Gênero & $54(48)$ \\
Homens & $58(52)$ \\
Mulheres & \\
Recidiva & $12(14,3)$ \\
Locorregional & $27(32,1)$ \\
Distância & $9(10,7)$ \\
Locorregional + Distância & $36(42,9)$ \\
Ausente & \\
Óbito & $45(40,2)$ \\
Doença neoplásica & $4 \quad(3,6)$ \\
Outras causas & \\
\hline Os dados são apresentados com número (percentual), média $\pm d p$ \\
(min - máx), ou mediana (amplitude interquartil p25-p75).
\end{tabular}




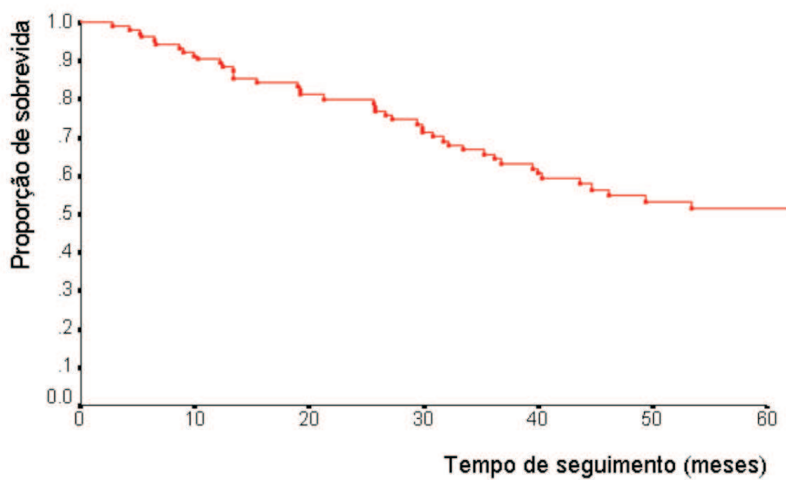

Figura 1 - Curva de sobrevida.

tidos (N2). Dezoito casos (16\%) eram tumores mucoprodutores. No momento do diagnóstico, 14 pacientes apresentavam metástases à distância, e 15 tiveram comprometimento de margem cirúrgica, totalizando 25 casos de cirurgias não-curativas (alguns com envolvimento simultâneo).

Cinqüenta e cinco pacientes encontravam-se nos estágios C1 e C2 de Dukes modificado por Astler-

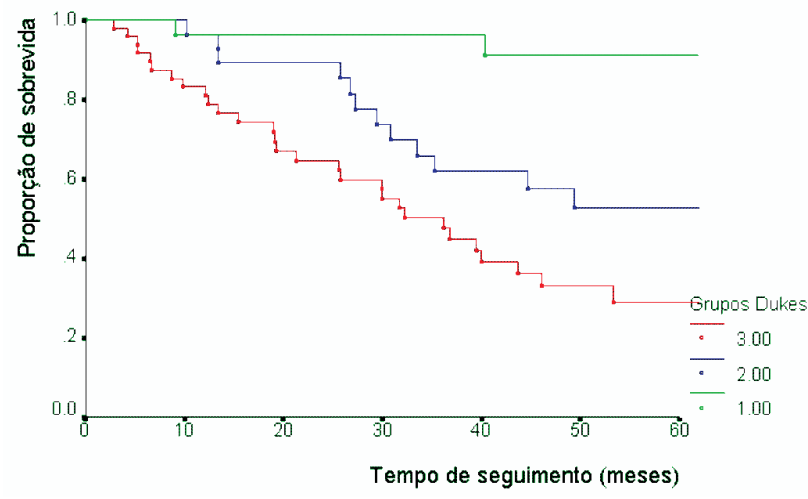

Figura 2 - Curvas de sobrevida pelos subgrupos de Dukes. $(1=A+B 1 ; 2=B 2+C 1 ; 3=C 2+D)$

Coller, correspondendo ao estágio III da classificação TNM, e 14 casos eram estágio D ou IV. Apenas 22\% dos pacientes ficaram classificados nos estágios mais precoces.

As variáveis: idade, sexo, níveis séricos de CEA pré-operatório, realização de tratamento complementar e produção de muco, não apresentaram significância estatística em relação ao óbito, na análise bivariada.

Tabela 2 - Características anatomopatológicas dos pacientes estudados.

\begin{tabular}{|c|c|c|}
\hline Característica & Medida d & scritiva \\
\hline Diferenciação tumoral & & \\
\hline Bem diferenciado & 21 & (19) \\
\hline Moderadamente diferenciado & 82 & (73) \\
\hline Pouco diferenciado & 9 & $(8)$ \\
\hline Grau de penetração na parede $(\mathrm{T})$ & & \\
\hline Submucosa (T1) & 4 & (4) \\
\hline Muscular (T2) & 31 & (28) \\
\hline Tecidos perirretais ou órgãos (T3 e T4) & 75 & (68) \\
\hline Comprometimento linfonodal $(\mathrm{N})$ & & \\
\hline Linfonodos negativos (N0) & 40 & $(37)$ \\
\hline 1 a 3 linfonodos positivos (N1) & 33 & $(31)$ \\
\hline 4 ou mais linfonodos positivos (N2) & 34 & (32) \\
\hline $\mathrm{N}^{\mathrm{o}}$ linfonodos isolados & & \\
\hline Total geral & 14,39 o 10,25 & $(0-48)$ \\
\hline Positivos & 6,85 o $\quad 7,89$ & $(1-39)$ \\
\hline$\%$ positividade & 42,02 & $(24,3-77,1)$ \\
\hline Metástases (M) & & \\
\hline Ausência de metástases (M0) & 98 & $(87)$ \\
\hline Presença de metástases (M1) & 14 & $(13)$ \\
\hline
\end{tabular}

Os dados são apresentados com número (percentual), média $\pm d p$ (min -máx), ou mediana (intervalo entre quartis p25-p75). \% positividade= $n^{o}$ linfonodos positivos $/ n^{o}$ total de linfonodos isolados. 
Tabela 3 - Resultados da análise bivariada - características clínicas em relação ao óbito.

\begin{tabular}{|c|c|c|c|c|c|c|}
\hline Variável & $\mathbf{n}$ & Óbito f & $\%$ & $\mathbf{R R}$ & IC $95 \%$ & $\mathbf{P}$ \\
\hline \multicolumn{7}{|l|}{ Idade (anos) } \\
\hline$=40$ & 11 & 5 & 45,5 & 1,00 & & \\
\hline$>40-60$ & 27 & 10 & 37,0 & 0,81 & $0,36-1,84$ & 0,969 \\
\hline$>60$ & 73 & 30 & 41,1 & 0,90 & $0,45-1,83$ & \\
\hline \multicolumn{7}{|l|}{ Gênero } \\
\hline Masculino & 54 & 21 & 38,9 & 0,92 & $0,59-1,45$ & 0,879 \\
\hline Feminino & 57 & 24 & 42,1 & & & \\
\hline \multicolumn{7}{|l|}{ Recidiva } \\
\hline Ausente & 36 & 0 & 0 & 1,00 & & \\
\hline Presente & 48 & 31 & 64,6 & 47,6 & $3,01-75,24$ & $<0,001$ \\
\hline Doença metastática & 14 & 13 & 92,9 & 66,6 & $4,22-1050,4$ & \\
\hline \multicolumn{7}{|c|}{ Tratamento complementar } \\
\hline $\mathrm{RT}+\mathrm{QT}$ & 7 & 2 & 28,6 & 1,00 & & \\
\hline RT & 9 & 3 & 33,3 & 1,17 & $0,26-5,19$ & 0,441 \\
\hline QT & 5 & 2 & 40,0 & 1,40 & $0,29-6,86$ & \\
\hline Ausente & 87 & 36 & 41,4 & 1,45 & $0,44-4,80$ & \\
\hline
\end{tabular}

Doença metastática = pacientes com doença disseminada no momento da cirurgia, não avaliáveis para recidiva.

Os pacientes que desenvolveram recidiva tumoral tiveram maior risco de óbito (Tabela $3 ; P<0,001$ ).

Os pacientes com tumores menos diferenciados $(P=0,009)$, com maior penetração na parede
$(P=0,013)$, com envolvimento linfonodal crescente $(\mathrm{N} 2>\mathrm{N} 1, P<0,001)$ e com metástases à distância no diagnóstico $(P<0,001)$ apresentaram maior risco de óbito (Tabela 4).

Tabela 4 - Resultados da análise bivariada - características anatomopatológicas em relação ao óbito.

\begin{tabular}{|c|c|c|c|c|c|c|}
\hline Variável & $\mathbf{n}$ & Óbito f & $\%$ & $\mathbf{R R}$ & IC $95 \%$ & $\mathbf{P}$ \\
\hline \multicolumn{7}{|c|}{ Diferenciação tumoral } \\
\hline Bem & 21 & 3 & 14,3 & 1,00 & & \\
\hline Moderadamente & 81 & 37 & 45,7 & 3,20 & $1,09-9,36$ & $0,009^{*}$ \\
\hline Pouco & 9 & 5 & 55,6 & 3,89 & $1,17-12,91$ & \\
\hline \multicolumn{7}{|c|}{ Classificação do tumor (T) } \\
\hline $\mathrm{T} 3 \mathrm{e} \mathrm{T} 4$ & 75 & 37 & 50,0 & 2,19 & $1,14-4,19$ & 0,013 \\
\hline $\mathrm{T} 1 \mathrm{e} \mathrm{T} 2$ & 35 & 8 & 22,9 & & & \\
\hline \multicolumn{7}{|l|}{ Linfonodos } \\
\hline Positivos & 66 & 36 & 54,5 & 3,12 & $1,53-6,33$ & $<0,001$ \\
\hline Negativos & 40 & 7 & 17,5 & & & \\
\hline \multicolumn{7}{|c|}{ Classificação dos linfonodos (N) } \\
\hline NO & 40 & 7 & 17,5 & 1,00 & & \\
\hline $\mathrm{N} 1$ & 31 & 15 & 48,4 & 2,76 & $1,29-5,94$ & $<0,001^{*}$ \\
\hline $\mathrm{N} 2$ & 35 & 21 & 60,0 & 3,43 & $1,66-7,08$ & \\
\hline \multicolumn{7}{|c|}{ Classificação das metástases (M) } \\
\hline M1 & 14 & 13 & 92,9 & 2,81 & $2,05-3,87$ & $<0,001$ \\
\hline M0 & 97 & 32 & 33,0 & & & \\
\hline
\end{tabular}

Bem = bem diferenciado; Moderadamente = moderadamente diferenciado; Pouco $=$ pouco diferenciado.

$* x^{2}$ de Mantel Haenszel para tendência linear. 
Da mesma forma, a mortalidade foi proporcional ao avanço dos estágios de Dukes modificado por Astler-Coller $(P<0,001)$, que se correlacionou fortemente com o sistema TNM (Tabela 5; o =0,91).

$\mathrm{Na}$ análise da mortalidade estratificada pelos subgrupos de Dukes, parece ter havido uma piora na sobrevida dentro de uma mesma categoria de Dukes, em relação aos tumores menos diferenciados, assim como houve discreta piora na sobrevida dos pacientes que desenvolveram recidiva em cada subgrupo de Dukes. Ambas as análises, contudo, não atingiram significância estatística.

A análise multivariada identificou a classificação de Dukes modificada por Astler-Coller e a diferenciação tumoral $(P=0,089)$, como fatores prognósticos independentes (Tabela 6).

Quando avaliadas as características isoladas do tumor (excluindo os estágios histopatológicos como um todo), o grau de penetração na parede intestinal
$(P=0,091)$ e o comprometimento linfonodal $(P<0,001)$ demonstraram ser fatores prognósticos independentes, aumentando o risco de óbito (Tabela 7). A faixa etária de 40 a 60 anos apresentou redução do risco que quase atingiu a significância estatística do modelo $(P=0,131)$, podendo indicar efeito protetor, ou maior risco para as faixas etárias mais jovens e mais idosas.

\section{DISCUSSÃO}

A média de idade e a distribuição por sexo mantiveram as características anteriormente descritas por Rosito et al. ${ }^{(13)}$,e correspondem à distribuição usual na literatura ${ }^{(2,14,15)}$. Embora não tenham sido relacionados à recorrência local em um estudo multicêntrico ${ }^{(16)}$, outros autores encontraram valor prognóstico independente para o sexo, com pior sobrevida entre os indivíduos do sexo masculino ${ }^{(12,17-19)}$. Em nossa experiência, nem a idade nem o sexo mostraram ser fatores prog-

Tabela 5 - Resultados da análise bivariada - classificações histopatológicas em relação ao óbito.

\begin{tabular}{lcrrrrr}
\hline Variável & $\mathbf{n}$ & Óbito f & \% & RR & IC 95\% & P \\
\hline Dukes & & & & & & \\
A + B1 & 28 & 2 & 7,1 & 1,00 & & \\
B2 + C1 & 31 & 13 & 41,9 & 5,87 & $1,45-23,77$ & $<0,001^{*}$ \\
C2 + D & 52 & 30 & 57,7 & 8,08 & $2,08-31,33$ & \\
Estágios TNM & & & & & & \\
I & 23 & 2 & 8,7 & 1,00 & & \\
II & 14 & 3 & 21,4 & 2,46 & $0,47-12,98$ & $<0,001^{*}$ \\
III & 54 & 25 & 46,3 & 5,32 & $1,37-20,64$ & \\
IV & 14 & 13 & 92,9 & 10,68 & $2,82-40,47$ & \\
\hline
\end{tabular}

$* x^{2}$ de Mantel Haenszel para tendência linear.

Tabela 6 - Modelo de Cox apresentando riscos relativos para ocorrência de óbito segundo subgrupos de Dukes modificada por Astler-Coller e diferenciação tumoral.

\begin{tabular}{lccccc}
\hline Variável & Coeficiente & EP & P & RR & IC 90\% \\
\hline Dukes & & & & & \\
A + B1 & - & - & - & - & \\
B2 + C1 & 1,625 & 0,776 & 0,036 & 5,08 & $1,42-18,20$ \\
C2 + D & 2,337 & 0,746 & 0,002 & 10,35 & $3,04-35,29$ \\
Diferenciação tumoral & & & & & \\
Bem diferenciado & - & - & - & - & \\
Moderadamente diferenciado & 0,888 & 0,619 & 0,152 & 2,43 & $0,87-6,73$ \\
Pouco diferenciado & 1,285 & 0,755 & 0,089 & 3,61 & $1,04-12,51$ \\
\hline
\end{tabular}


Tabela 7 - Modelo de Cox apresentando riscos relativos para ocorrência de óbito segundo variáveis de interesse.

\begin{tabular}{|c|c|c|c|c|c|}
\hline Variável & Coeficiente & EP & $\mathbf{P}$ & $\mathbf{R R}$ & IC $90 \%$ \\
\hline \multicolumn{6}{|l|}{ Comprometimento LN } \\
\hline No & - & - & - & - & \\
\hline N1 & 0,661 & 0,531 & 0,213 & 1,94 & $0,81-4,64$ \\
\hline N2 & 1,77 & 0,511 & $<0,001$ & 5,88 & $2,54-13,64$ \\
\hline \multicolumn{6}{|l|}{ Diferenciação tumoral } \\
\hline Bem diferenciado & - & - & - & - & \\
\hline Moderadamente diferenciado & 0,758 & 0,641 & 0,237 & 2,13 & $0,74-6,12$ \\
\hline Pouco diferenciado & 0,685 & 0,821 & 0,404 & 1,98 & $0,51-7,65$ \\
\hline Penetração na parede retal & 0,813 & 0,482 & 0,091 & 2,25 & $1,02-4,98$ \\
\hline Produção de muco & $-0,489$ & 0,608 & 0,421 & 0,61 & $0,22-1,66$ \\
\hline Gênero & 0,353 & 0,380 & 0,353 & 1,42 & $0,76-2,66$ \\
\hline \multicolumn{6}{|l|}{ Grupos etários* } \\
\hline Abaixo de 40 & - & - & - & - & \\
\hline 40 a 60 & $-1,012$ & 0,671 & 0,131 & 0,36 & $0,12-1,09$ \\
\hline Acima de 60 & 0,016 & 0,633 & 0,980 & 1,01 & $0,36-2,88$ \\
\hline CEA sérico & 0,0004 & 0,003 & 0,892 & 1,00 & $0,99-1,00$ \\
\hline
\end{tabular}

$L N=$ linfonodos.

* Grupos etários em anos.

nósticos independentes para a sobrevida. O possível efeito protetor da faixa etária de 40 a 60 anos pode não corresponder à realidade. No entanto, Nan et al. ${ }^{(20)}$ encontraram pior sobrevida no grupo de idosos acima de 75 anos portadores de câncer colorretal, tendo relacionado o achado ao diagnóstico tardio. Da mesma forma, também atribuindo ao retardo no diagnóstico, com $66 \%$ dos pacientes em estágios III e IV da doença-porém associado com tumores mais agressivos, o grupo etário mais jovem (abaixo de 40 anos) também tem sido relacionado a piores prognósticos ${ }^{(2,12,21,22)}$, com altas taxas de mortalidade (25\% num seguimento médio de 26 meses) ${ }^{(23)}$. No entanto, parece que esse efeito é minimizado quando a análise de sobrevida é ajustada de acordo com o estágio do tumor ${ }^{(12)}$. Nesta casuística, necessitaríamos de um número maior de pacientes para confirmar tal achado.

No momento da cirurgia, $13 \%$ dos pacientes apresentavam metástases à distância. $\mathrm{O}$ índice de cirurgias não-curativas foi de $22,5 \%$, considerando esses casos com doença metastática $(n=14)$ e os pacientes com comprometimento do limite radial das margens cirúrgicas $(n=15$; alguns casos apresentaram envolvimento simultâneo). Estes casos são invariavelmente relacionados a piores prognósticos ${ }^{(8,12,24,25)}$, fato este também observado nesta série. Em outras revisões, em torno de $25 \%$ dos pacientes já apresentavam envolvimento metastático à distância no momento do diagnóstico ${ }^{(26)}$.

O índice de recidiva locorregional isolada foi de $14 \%$. Outros $10,7 \%$ desenvolveram recidiva locorregional associada a metástases à distância, totalizando $24,7 \%$ de recorrência tumoral local. Pinho et al. ${ }^{(7)}$, em um levantamento prospectivo de 97 pacientes com câncer colorretal submetidos à cirurgia com intenção curativa, encontraram um percentual de $12,5 \%$. $\mathrm{Na}$ casuística anterior do Serviço, a recidiva locorregional ocorreu em $20 \%$ dos $\operatorname{casos}^{(13)}$. As taxas de recorrência local, no mundo, variam de 20 a $45 \%^{(10,16,27-29)}$. Sabe-se que os melhores índices obtidos até hoje foram de 2,6\% com a ressecção total do mesorreto $^{(10,30)}$. Resultados semelhantes $\left(3,8 \%{ }^{(31)} \mathrm{e}\right.$ $4,7 \%^{(32)}$ ) têm sido obtidos em nosso meio com o auxílio de terapia complementar pré-operatória. Os casos de recidiva, em nossa casuística, tiveram uma mortalidade significativamente maior. Na literatura, a sobrevida em 5 anos após ressecção da recidiva não chega a $30 \%$, comparável aos casos de doença metastática ${ }^{(33)}$. A ocorrência de recidiva aumentou progressivamente à medida que avançaram os estágios histopatológicos 
da doença. Esse achado também foi observado por outros autores ${ }^{(16)}$. Na análise estratificada, no entanto, somente no subgrupo de Dukes de pior prognóstico, os pacientes com metástases à distância e recidiva apresentaram risco significativo de óbito em relação àqueles sem recidiva.

No período de seguimento (mediano de 35,27 meses), $44 \%$ dos pacientes foram a óbito, sendo $40 \%$ da mortalidade relacionada à neoplasia. Esses dados correspondem aos achados da literatura ${ }^{(2,8,17,34)}$. O tempo mediano de sobrevida relacionada à neoplasia foi de 71,8 meses. A sobrevida global em 5 anos foi de $51 \%$. A proporção de sobrevida piorou significativamente à medida que avançaram os estágios de Dukes (subgrupo 1, 91\%; subgrupo 2, 57\%; e subgrupo 3,39\%) - no período de 40 meses, tendo em vista que o primeiro grupo não alcançou $50 \%$ de mortalidade no tempo mediano de sobrevida geral (71,83 meses). Pinho et al. ${ }^{(7)}$ encontraram uma sobrevida em 5 anos de $52 \%$, sendo $89 \%$ para pacientes estágio $1,70 \%$ para pacientes estágio 2 e $20 \%$ para os pacientes estágio 3 . No relato de Norstein et al. ${ }^{(16)}$, a sobrevida em 5 anos foi de $55,2 \%$, também piorando quanto mais avançado o estágio da doença (77\% Dukes A; 48\% Dukes B; e $35 \%$ Dukes C). Da mesma forma, Hermanek et al. ${ }^{(27)}$ observaram uma sobrevida relacionada à doença neoplásica de $66 \%$, com uma variabilidade proporcional aos estágios de TNM: estágio I, de 89 a $100 \%$ entre os centros; estágio II, de 58 a 92\%; e estágio III, de 13 a $69 \%$. Nan et al. ${ }^{(20)}$ também encontraram sobrevida relacionada aos estágios de Dukes, que demonstrou ser fator prognóstico independente na análise multivariada $^{(20)}$.

O valor da dosagem pré-operatória de CEA sérico na avaliação prognóstica dos pacientes tem sido amplamente discutido ${ }^{(8,12,24,35)}$. Embora níveis elevados estejam sendo relacionados, a maior risco de recidiva e piora na sobrevida por alguns autores ${ }^{(12,17,36)}$, essa variável não foi identificada como um fator prognóstico independente na análise multivariada ${ }^{(17)}$. Contudo, tem sido postulado que uma dosagem pré-operatória elevada pode estar relacionada a recorrência e piora na sobrevida ${ }^{(12)}$. Atualmente, a dosagem pré-operatória de CEA tem sido usada como referência para o seguimento do paciente ${ }^{(17)}$. O uso da dosagem isolada de CEA para monitorar resposta terapêutica não está sendo recomendado ${ }^{(8)}$, mas a AJCC recomenda a inclusão desta característica em uma subcategoria do sistema TNM, como marcador prognóstico ${ }^{(24,35)}$. Em nosso estudo, a dosagem sérica do CEA pré-operatório não demonstrou impacto na sobrevida dos pacientes, embora se possa considerar que o tamanho amostral seja modesto para esta avaliação.

$\mathrm{O}$ adenocarcinoma moderadamente diferenciado foi o tipo histológico mais freqüente, em $73 \%$ dos casos, seguido de $18 \%$ de lesões bem diferenciadas e 9 casos de adenocarcinoma pouco diferenciado (8\%). Esses percentuais são semelhantes aos encontrados na literatura ${ }^{(15,37)}$, e aos observados previamente em nosso Serviço ( $20 \%$ bem diferenciados, $65 \%$ moderadamente diferenciados e $3,4 \%$, pouco diferenciados $11,6 \%$ foram considerados indiferenciados) $)^{(13)}$. $\mathrm{O}$ risco de óbito foi três vezes maior entre os tumores menos diferenciados $(P=0,009)$, na análise bivariada. Apesar disso, a análise estratificada não detectou efeito da diferenciação tumoral, possivelmente porque o tamanho amostral de cada subgrupo era pequeno. Já no modelo de Cox, esse efeito se manifestou em relação ao estrato dos tumores pouco diferenciados, quando comparado aos bem diferenciados, sendo independente do efeito dos estágios de Dukes. Os tumores menos diferenciados têm sido diretamente relacionados à recorrência local ${ }^{(38)} \mathrm{e}$ à piora na sobrevida ${ }^{(18-20)}$. Contudo, resultados conflitantes vêm sendo encontrados, possivelmente pelos complexos critérios de avaliação, que não são padronizados ${ }^{(18,20,34)}$. Entre 24 estudos realizados com análise multivariada, 10 demonstraram valor prognóstico independente, mas, em comparação com o estágio do tumor, o grau de diferenciação não acrescentou nenhuma vantagem significativa na previsão da sobrevida ${ }^{(17)}$. Em outro estudo, foi considerado fator prognóstico independente, juntamente com o estágio do tumor ${ }^{(20)}$.

Em 18 casos, os tumores eram produtores de muco. Essa característica não apresentou influência significativa na sobrevida, confirmando os achados de Rawet ${ }^{(18)}$ e Ratto et al. ${ }^{(17)}$ Contudo, a análise histopatológica de um tumor demonstrando áreas mucinosas ou células em anel de sinete tem levado à identificação de tumores mais agressivos, com pior potencial prognóstico ${ }^{(12)}$. No entanto, a piora do prognóstico parece ter sido atribuída ao estágio avançado da doença ${ }^{(17)}$.

A maior parte das lesões estendia-se até os tecidos perirretais $(68,2 \%)$. Os pacientes com tumores de maior grau de penetração na parede (T3 e T4) apresentaram o dobro do risco de óbito em relação aos menos avançados (T1 e T2). O grau de invasão tumoral 
provou ter relação direta com o prognóstico ${ }^{(24,25)}$. Cinquienta e cinco pacientes pertenciam aos estágios $\mathrm{C} 1 \mathrm{e}$ C2 de Dukes modificado por Astler-Coller (TNM III). Pouco mais de $20 \%$ dos casos ficaram classificados nos estágios mais precoces. Essa observação vai ao encontro da experiência comum de diagnósticos tardi$\mathrm{os}^{(22,23)}$, e deve reforçar a importância dos programas de prevenção e detecção precoces, uma vez que os tumores de reto médio e distal encontram-se acessíveis ao diagnóstico e ao estadiamento em exame clínico de rotina ${ }^{(39)}$.

A média (dp) de linfonodos isolados da peça cirúrgica foi de $14,4( \pm 10,25)$ linfonodos. A recomendação da $\mathrm{AJCC}^{(8)}$ é de que no mínimo 12 linfonodos sejam isolados da peça cirúrgica para a determinação confiável do nível de envolvimento linfonodal ${ }^{(8)}$, o que tem demonstrado influência negativa no prognósti$\mathrm{co}^{(12,20,24,40,41)}$. O número de linfonodos positivos variou de 1 a 39, com média (dp) de 6,8 $( \pm 7,9)$ linfonodos, correspondendo a mais de $60 \%$ dos casos com envolvimento linfonodal. Os pacientes com linfonodos positivos apresentaram risco de óbito três vezes maior, sendo que a quantificação dos linfonodos comprometidos demonstrou valor prognóstico significativo, corroborando achados de outros autores ${ }^{(15,17,18,34,41)}$. Cinco estudos investigaram o número de linfonodos com comprometimento metastático para determinação prognóstica; apenas dois destes mostraram valor preditivo adequado para este parâmetro no prognósti- $\mathrm{co}^{(17)}$. Esses dados contrastantes são possivelmente consequientes às variações entre técnicas histológicas, que podem subestadiar os tumores. A AJCC recomenda inclusão do envolvimento linfonodal metastático na categoria I, de fatores definidamente influentes no prognóstico ${ }^{(24)}$.

\section{CONCLUSÕES}

Os fatores que demonstraram efeito prognóstico independente nesta casuística foram: diferenciação tumoral, grau de penetração do tumor na parede e comprometimento linfonodal, além da classificação histopatológica propriamente dita.

A sobrevida relacionada à neoplasia dos pacientes com tumor de reto médio e distal foi de $51 \%$ em cinco anos. Observou-se uma piora significativa da recidiva tumoral e da sobrevida à medida que avançaram os estágios da doença. Os achados deste estudo estão de acordo com a literatura: apesar das limitações, os níveis dos sistemas de classificação histopatológica dos tumores ainda são os critérios prognósticos disponíveis. Estas conclusões devem ser reforçadas em estudos com maior número de pacientes.

\section{AGRADECIMENTO}

Os autores agradecem a Mario Wagner, PhD. pelo auxílio na análise estatística.

ABSTRACT: The objective of this study was to evaluate survival and clinicopathological factors in rectal adenocarcinoma, the records of 112 patients were reviewed for: age, gender, serum level of CEA, surgery curability, follow-up, recurrence, survival and tumor histopathology. Kaplan-Meyer curves were used to analyze survival. Statistical significance in bivariate and stratified analysis was set at $\boldsymbol{P}<\mathbf{0 . 0 5}$. In the multivariate model, a $90 \%$ confidence interval was considered significant. Median follow-up was 35 (14 - 57) months. Five-year survival rate was $51 \%$. Sixty-four patients (57\%) had recurrence; 45 (40\%) died from neoplasia, $68 \%$ tumors extended to perirectal tissues and 67 had positive lymph nodes (30\% each, N1 and N2). Fourteen patients were Dukes $D$ stage; 55 were $\mathrm{C} 1$ and $\mathrm{C} 2$; 15 were $\mathrm{B2}$; and 28 were $\mathrm{B} 1$ and $\mathrm{A}$. Death increased significantly with tumor progression stages $(P<0.001)$, tumor depth $(P=0.013)$ and grade $(P=0.009)$, lymph node involvement $(\mathrm{N} 2>\mathrm{N} 1, P<0.001)$ and recurrence $(P<0.001)$. Independent prognostic factors were Dukes stages and tumor grade $(P=0.089)$, as well as depth of invasion and lymph node involvement when Dukes staging was excluded $(P=0.091$ and $<0.001)$. Besides tumor grade, the prognostic factors identified meet classification levels in current staging systems.

Key words: Rectal adenocarcinoma, prognostic factors, histopathological staging, recurrence, survival.

\section{REFERÊNCIAS}

1. Ky AJ, Sung MW, Milsom JW. Research in colon and rectal cancer, with an emphasis on surgical progress. Dis Colon Rectum 1999; 42(11): 1369-1380.
2. Williams NS. Colorectal cancer: epidemiology, aetiology, pathology, clinical features and diagnosis. In: Keighley MR, Williams NS, eds. Surgery of the anus, rectum and colon. London: WB Saunders; 1993. p. $830-885$. 
3. Santos JCM Jr. Tratamento coadjuvante do câncer do reto: I Radioterapia pré-operatória. Rev Bras Coloproct 1997; 17(1): 69-73.

4. Wilmink AB. Overview of the epidemiology of colorectal cancer. Dis Colon Rectum 1997; 40(4): 483-493.

5. European School of Oncology. Task Force Preview, Geneva, Switzerland. Issue 2, Sept 1997.

6. Brasil, Ministério da Saúde, Instituto Nacional do Câncer, Coordenadoria de Programas de Controle do Câncer. O problema do câncer no Brasil. $4^{\mathrm{a}}$ ed. Rio de Janeiro: INCa/ ProOnco; 1997.

7. Pinho MSL, Ferreira LC, Kleinubing Jr. H. Tratamento cirúrgico do câncer colorretal: resultados a longo prazo e análise da qualidade. Rev Bras Coloproct 2006; 26(4): 422-429.

8. National Cancer Institute, PDQ. Rectal Cancer. 2007; 1-27. Available: http://www.cancer.gov

9. Pinho MSL. Marcadores moleculares como fatores prognósticos do câncer colorretal. Rev Bras Coloproct 1999; 19 (2): 130-132.

10. MacFarlane JK, Ryall RD, Heald RJ. Mesorectal excision for rectal cancer. Lancet 1993; 341(8843): 457-460.

11. Macdonald JS. Adjuvant therapy of colon cancer. CA Cancer J Clin 1999; 49(4): 202-219.

12. Department of pathology, SGPGIMS. Prognostic factors predicting outcome in colorectal cancers. On-line seminars and tutorials. Lucknow, India. 2007; 1-6. Available: http:// www.sgpgi.ac.in/path/seminars.

13. Rosito MA, Müssnich JFX, Gus PG, Weindorfer M, Schaefer LG, Pinheiro PM. Amputação abdomino-perineal em adenocarcinoma de reto. II levantamento do HCPA. Rev AMRIGS 1995; 39: 200-204.

14. Dias GB, Bauermann FB, Linhares EL, Mercado C, Moreira LF. Estudo retrospectivo do índice de mortalidade por câncer de cólon e de reto em Porto Alegre e no Rio Grande do Sul. Proc XV JACAD 1997; 15: TL038-29.

15. Gordon PH. Malignant neoplasms of the colon. In: Gordon $\mathrm{PH}$, Nivatvongs S, eds. Principles and practice of surgery for the colon, rectum and anus. St. Louis: Quality Medical; 1992. p. 501-565.

16. Norstein J, Langmark F. Results of rectal cancer treatment: a national experience. In: Søreide O, Norstein J, eds. Rectal cancer surgery. Optimisation - Standardisation Documentation. Oslo: Springer; 1997. p. 17-28.

17. Ratto C, Sofo L, Ippoliti M, Merico M, Doglietto GB, Crucitti F. Prognostic factors in colorectal cancer: literature review for clinical application. Dis Colon Rectum 1998; 41(8): 1033-1049.

18. Rawet V. Carcinoma colorretal: estadiamento e parâmetros prognósticos [tese]. São Paulo: Universidade de São Paulo; 1998.

19. Bokey EL, Chapuis PH, Dent OF, Newland RC, Koorey SG, Zelas PJ, et al. Factors affecting survival after excision of the rectum for cancer: a multivariate analysis. Dis Colon Rectum 1997; 40(1): 3-10.
20. Nan KJ, Qin HX, Yang G. Prognostic factors in 165 elderly colorectal cancer patients. World J Gastroenterol. 2003; 9(10): 2207-2210.

21. Jessup JM, Menck HR, Fremgen A, Winchester DP. Diagnosing colorectal carcinoma: clinical and molecular approaches. CA Cancer J Clin 1997; 47(2): 70-92.

22. Carneiro Neto JD, Barreto JBP, Freitas NS, Queiroz MA. Câncer colorretal: características clínicas e anatomopatológicas em pacientes com idade inferior a 40 anos. Rev Bras Coloproct, 2006; 26(4): 430-435.

23. Monteiro EP, Salem JB, Taglietti EM, Albuquerque IC, Formiga GJS. Neoplasia colorretal até 40 anos - experiência em cinco anos. Rev Bras Coloproct. 2006; 26(2):156-161.

24. Compton CC, Fielding LP, Burgart LJ, Conley B, Cooper HS, Hamilton SR, et al. Prognostic factors in colorectal cancer. Arch Pathol Lab Med. 2000; 124(7): 979-994.

25. Wood CB, Gillis CR, Hole D, Malcolm AJ, Blumgart LH. Local tumour invasion as a prognostic factor in colorectal cancer. Br J Surg.1981; 68(5): 326-328.

26. Santos JCM Jr. Tratamento coadjuvante do câncer do reto: II - Quimioterapia. Rev Bras Coloproct 1997; 17(2): 143-149.

27. Hermanek P. Impact of surgeon's technique on outcome after treatment of rectal carcinoma. Dis Colon Rectum 1999; 42(5): 559-562.

28. Moriya Y, Hojo K, Sawada T, Koyama Y. Significance of lateral node dissection for advanced rectal carcinoma at or below the peritoneal reflection. Dis Colon Rectum 1989; 32(4): 307315.

29. Moreira LF, Hizuta H, Iwagaki H, Tanaka N, Orita K. Lateral lymph node dissection for rectal carcinoma below the peritoneal reflection. Br J Surg 1994; 81(2): 293-296.

30. Heald RJ, Smedh RK, Kald A, Sexton R, Moran BJ. Abdominoperineal excision of the rectum - an endangered operation. Norman Nigro Lectureship. Dis Colon Rectum 1997; 40(7): 747-751.

31. Reis Neto JA, Cordeiro F, Reis JA Jr, Kagohara O, Simões Neto J. Laparoscopic total mesorectum excision. In: Reis Neto JA, ed. New trends in coloproctology. Campinas: Revinter; 2000. p. 497-504.

32. Habr-Gama A, Souza PM, Ribeiro U Jr., Campos F, Sousa AH Jr., Nadalin W, Gansl R, et al. Low rectal cancer: impact of preoperative radiation and chemotherapy on surgical treatment. "Hospital das Clínicas" of the University of São Paulo Medical School experience. In: Reis Neto JA, ed. New trends in coloproctology. Campinas: Revinter; 2000. p. 423-429.

33. Bozzetti F, Bertario L, Rossetti C, Gennari L, Andreola S, Baratti D, et al. Surgical treatment of locally recurrent rectal carcinoma. Dis Colon Rectum 1997; 40(12): 1421-1424.

34. Søreide O, Norstein J, Fielding P, Silen W. International standardization and documentation of the treatment of rectal cancer. In: Søreide O, Norstein J, eds. Rectal cancer surgery. Optimisation - Standardisation - Documentation. Oslo: Springer; 1997. p. 405-446. 
35. Compton C, Fenoglio-Preiser CM, Pettigrew N, Fielding LP. American Joint Committee on Cancer Prognostic factors consensus conference: Colorectal Working Group. Cancer 2000; 88(7): 1739-1757.

36. Kim JC, Han MS, Lee HK, Kim WS, Park SK, Park KC, et al. Distribution of carcinoembryonic antigen and biologic behavior in colorectal carcinoma. Dis Colon Rectum 1999; 42(5):640648.

37. Mulcahy HE, Toner M, Patchett SE, Daly L, O’Donoghue DP. Identifying stage $\mathrm{B}$ colorectal cancer patients at high risk of tumor recurrence and death. Dis Colon Rectum 1997; 40(3): 326-331.

38. Reis Neto JA, Quilici FA, Cordeiro F, Ciquini S, Reis JA Jr. Pre-operative radiotherapy in rectal cancer: evaluation of irradiation effects on cellular undifferentiation and its influence on prognosis. Hepatogastroenterology 1999; 46(29): 28252830 .
39. Jucá MJ, Matos D, D’Ippolito G. Estudo da validação do exame digital do reto no estadiamento clínico pré-operatório dos adenocarcinomas tocáveis de reto. Rev Bras Coloproct 1999; 19(4):248-258.

40. Chok KS, Law WL. Prognostic factors affecting survival and recurrence of patients with pT1 and pT2 colorectal cancer. World J Surg. 2007; 31(7): 1485-90. Epub 2007 May 18.

41. Hetnal M, Malecki K, Korzeniowski S, Zemelka T. Postoperative chemoradiotherapy in patients with rectal cancer, prognostic factors for disease control and survival. J Clin Oncol. 2006; 24(18S): 13575.

\section{Endereço para correspondência:} HELOISA GUEDES MÜSSNICH

Rua Ramiro Barcelos, 910/ conj. 1001

CEP 90035-001 - Porto Alegre - RS - Brasil

Fone/Fax: (51) 3311-7608

E-mail: lolomuss@brturbo.com.br 